\title{
Bicontinuous cubic phases in biological and artificial self-assembled systems
}

\author{
Congcong Cui ${ }^{1}$, Yuru Deng ${ }^{2^{*}}$ and Lu Han ${ }^{1 *}$
}

\begin{abstract}
Nature has created innumerable life forms with miraculous hierarchical structures and morphologies that are optimized for different life events through evolution over billions of years. Bicontinuous cubic structures, which are often described by triply periodic minimal surfaces (TPMSs) and their constant mean curvature (CMC)/parallel surface companions, are of special interest to various research fields because of their complex form with unique physical functionalities. This has prompted the scientific community to fully understand the formation, structure, and properties of these materials. In this review, we summarize and discuss the formation mechanism and relationships of the relevant biological structures and the artificial self-assembly systems. These structures can be formed through biological processes with amazing regulation across a great length scales; nevertheless, artificial construction normally produces the structure corresponding to the molecular size and shape. Notably, the block copolymeric system is considered to be an applicable and attractive model system for the study of biological systems due to their versatile design and rich phase behavior. Some of the phenomena found in these two systems are compared and discussed, and this information may provide new ideas for a comprehensive understanding of the relationship between molecular shape and resulting interface curvature and the selfassembly process in living organisms. We argue that the copolymeric system may serve as a model to understand these biological systems and could encourage additional studies of artificial self-assembly and the creation of new functional materials.
\end{abstract}

Keywords: triply periodic minimal surface, self-assembly, cubic membrane, lyotropic liquid crystal, block copolymer

\section{INTRODUCTION}

Triply periodic minimal surfaces (TPMSs) are smooth and nonintersecting curved surfaces that have three- dimensional (3D) periodicity and vanishing mean curvature $H$ everywhere [1,2]. This balanced continuous surface separates the space into two intertwining labyrinths known as bicontinuous structures. A skeletal graph can be used for their structural visualization by modeling the centers of the labyrinths using simple rods connecting corresponding nodes. The most common and important TPMSs are the Schwarz primitive (P), Schwarz diamond (D) and Schoen gyroid (G) surface structures. The P surface is also known as "plumber's nightmare", which has two interpenetrating cubic networks with six-fold connectivity with space group Im-3m (No. 229). The D surface separates two diamond networks with four-fold connectivity with space group $P n-3 m$ (No. 224). The most popular G surface has three-fold connectivity with neither a straight line nor a mirror plane in the structure. Although various screw axes from different directions exist, the gyroid structure is achiral due to the double networks with opposite handedness, and its space group is $I a-3 d$ (No. 230). On the other hand, a family of constant mean curvature (CMC) surface with $H \neq 0$ and parallel surfaces shifted off the TPMS along the surface normal with fixed distance can be generated from their corresponding TPMS families.

The TPMSs and their corresponding $\mathrm{CMC} /$ parallel surface companions are attractive not only because of their complexity and geometrical beauty but also due to their unique physical properties. These surfaces have been used to model various crystalline structures both in nature and in artificial systems. Examples include the echinoderm skeletons [3,4], cell endomembrane systems [5,6], butterfly wing scales and exoskeletons of beetles and weevils [7-11], the retinal cone photoreceptor cells of tree shrews [12], lyotropic liquid crystals (LLCs) [13-18], and block copolymer self-assemblies [19-25]. These surfaces can also be used to model zeolites $[26,27]$ and periodic

\footnotetext{
${ }^{1}$ School of Chemical Science and Engineering, Tongji University, Shanghai 200092, China

${ }^{2}$ Wenzhou Institute, University of Chinese Academy of Sciences, Wenzhou 325001, China

* Corresponding authors (emails: luhan@tongji.edu.cn (Han L); dengyr@wibe.ac.cn (Deng Y))
} 
zero potential surfaces in atomistic crystals [28]. Such structures and their analogues have been found to possess unique functions, such as structural colors [7-11], unique mechanical and electronic properties $[29,30]$, membrane protein crystallization and drug delivery [31-33]. Additionally, it is noteworthy that their formation mechanism might have much in common with cell membrane-related processes [34,35].

Among the biological structures, the cubic cell membranes with TPMSs and related CMC surfaces are of great interest. These structures have been frequently discovered in numerous biomembranes under various conditions, such as the plasma membrane, endoplasmic reticulum, nuclear envelope, chloroplasts, inner mitochondrial membrane [12]. However, the formation mechanism and the corresponding biological functions of such delicate structures are still unknown, largely due to the difficulties in revealing their formation process in the complex biological environment and their structural characterizations due to the soft nature of these systems. In addition, in some of these structures, the unit cell parameters can be up to several hundred nanometers in order to show the corresponding optical functionalities $[7,11,12,36]$. These phenomena pose challenges to the research field in its attempts to fully understand the detailed structure, structural relationship, and the formation process of these cubic membranes.

On the other hand, the liquid crystal (LC) systems and the microphase separation of the block copolymers have been used as a versatile tool for fabricating periodic structures due to the high flexibility of molecular design (Fig. 1). However, the artificial fabrication only achieves basic structural categories that natural systems have evolved, and the typical unit cell parameter is normally limited to a range of several nanometers for LC systems and 5-100 nm for block copolymer systems [25,37]. Regarding the artificial fabrication of these structures, it has been generally accepted that the structures formed in amphiphilic systems can be understood by cross correlation between the interface curvatures and the geometrical characteristics of the chemicals employed. Recently, new developments in the microphase separation of copolymeric systems have brought new insight into the formation of hyperbolic surface structures [38-40]. The addition of solvent enables the solubilization of the block copolymer and the swelling of the whole system, which forms structures with different Gaussian curvatures. This self-assembly process presents a challenge to the correlation between molecular shape and the interfacial curvature, which may shed light on the formation

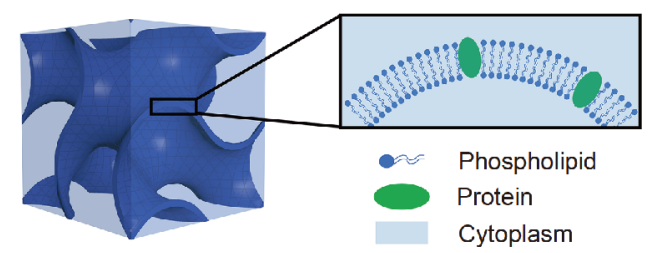

Biological membrane systems
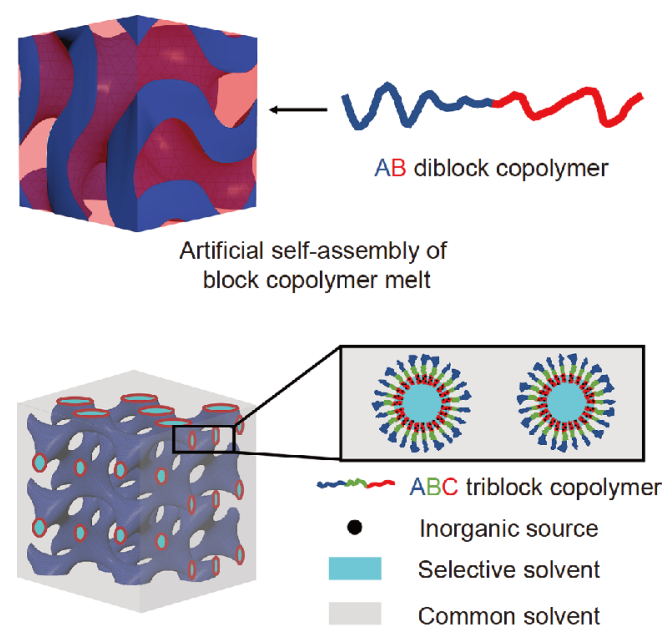

Artificial self-assembly of block copolymer with solvents

Figure 1 Schematic diagram of the molecule packing in biological cubic membranes and the self-assembly of block copolymers.

mechanism of these structures with hyperbolic surfaces, in addition to our understanding in biology-derived relevant structures [41].

This review article introduces three aspects of understanding the formation and structures of biological and artificial self-assembly systems. First, we introduce several representative cubic membrane structures in vivo. We then review the self-assembly of block copolymers into cubic phases, from pure block copolymer melts to complex systems involving inorganics. Finally, we compare the biological and copolymeric systems with respect to their formation mechanism and structural relationship. By highlighting the similarities and differences in biological and artificial self-assembly systems, we demonstrate that amphiphilic self-assembly may be inspired by biological systems. More importantly, artificial self-assembly may provide a good model system for understanding the formation mechanism of these delicate structures in nature.

\section{BIOLOGICAL BICONTINUOUS CUBIC STRUCTURES}

In biological systems, highly organized and delicate 
structures are amazingly optimized to serve biological function(s) (if there is), which may be far beyond the capabilities of artificial fabrication. In order to adapt to external environmental changes, organisms have evolved to form a series of complex structures with corresponding functions at microscopic, mesoscopic, and macroscopic scales. Biological membranes that are essential for living cells are considered to be one of the most attractive assemblies in nature. They define the interface between the outside and inside of cells and organelles, enabling different biochemical reactions to occur in different intracellular compartments. These membranes are often formed by steroid lipids with embedded membrane proteins, which, through noncovalent forces, form a phospholipid-based bilayer matrix with a thickness of several nanometers [42-49]. Phospholipid molecules contain a hydrophilic polar head group and one or two hydrophobic fatty acyl chains. The self-assembly of the membranes relies primarily on the hydrophobic interactions of lipid molecules and proteins. The hydrophobic core can form spontaneously in water, forming the basis of the biological membrane self-assembly system [49-52]. Among these, cubic membranes, discovered in various intracellular membranes of different life forms, deserve specific attention. Planar biological membranes can fold into cubic membranes, particularly in cells in response to starvation or oxidative stress, viral infection, abnormal protein expression, etc. [53-56]

The structure of cubic membranes is similar to that of bicontinuous cubic phases. They are produced in living organisms and rapidly disappear with apoptosis, which makes their structural study by scattering very difficult. Therefore, their structures have mainly been revealed by transmission electron microscopy (TEM), especially cryoTEM [57,58]. Landh [59] first investigated the cell membrane structures using TEM and computer simulation of TEM images and discussed the possible physiological significance of these structures. By comparing a large number of cross sections of cytomembranes with different thicknesses, the $\mathrm{D}$ surface structure was identified in the prolamellar body (PLB, etioplast) of plants. This demonstrated the existence of TPMSs in biological membranes. This technique can be extended to examine other soft matter systems. As bicontinuous cubic phase structures can be generated by level surface approximation, a computer-generated two-demensional (2D) projection library has been used to match the TEM images to further confirm the structure of cubic membranes $[6,55,59,60]$.

The chloroplast has a thylakoid membrane with a la- mellar stack structure, which is present in all the green organs of plants and is formed by etioplast differentiation [61]. Etioplasts have a unique intimal system consisting of a paracrystalline PLB with a cubic phase structure and interconnected lamellar prothylakoids [62]. The building unit is usually a 4 -arm (diamond surface) or a small amount of a 6-arm (primitive surface) [62,63]. At a biochemical level, the main feature of etioplasts is the lack of chlorophyll. However, it accumulates enzymes and precursors that produce chlorophyll under light conditions. PLBs disappear in chlorophyll-rich chloroplasts that are fully differentiated from etioplasts [64-66]. In addition, chloroplasts can also produce PLBs reversibly in dark or low light intensity, which disappear after light is restored [66]. Compared to PLBs in etioplasts, the PLBs in chloroplasts are usually smaller in size and larger in number [67,68]. Light stimulation, chlorophyll, and membrane proteins involved in photosynthesis control the transition between PLBs with a bicontinuous cubic phase and lamellar structures. It is worth noting that the cubic membrane is asymmetric on both sides, forming the stroma of the etioplast and the PLB lumen $[63,69,70]$.

Another important finding involves the cubic membrane structure in amoeba (Chaos carolinense) mitochondria. Deng et al. [55,58,71] studied the cubic membrane structure in detail by conventional scanning electron microscopy (SEM), TEM and electron microscopic tomography under starvation conditions and identified the isolation buffer conditions that preserve cubic membrane morphology in vitro (Fig. 2a, b). Cubic membranes have a large lattice parameter and pore size, which is usually 10 -fold larger than that of the cubic phase structure of the lipid molecule self-assembly system [72]. Formation of cubic membrane structures in amoeba mitochondria was found to be dependent on diet conditions. Amoeba Chaos mitochondrial cubic structures only appeared in the absence of their natural food source (Paramecium) and disappeared in the presence of Paramecium. Moreover, these mitochondria with cubic structures could escape from autophagy to maintain their normal function of producing adenosine triphosphate (ATP) during long-term starvation periods [73]. Almsherqi et al. [60] and Goldsmith et al. [74] also used the same method to determine the severe acute respiratory syndrome (SARS) viral induction of cubic membrane formation (Fig. 2c, d).

Another example is lung surfactant, an aqueous lipidprotein system on the alveolar surface of the mammalian lung that is necessary for normal breathing. Early studies of rabbit lung surfactant revealed a tetragonal lattice with 


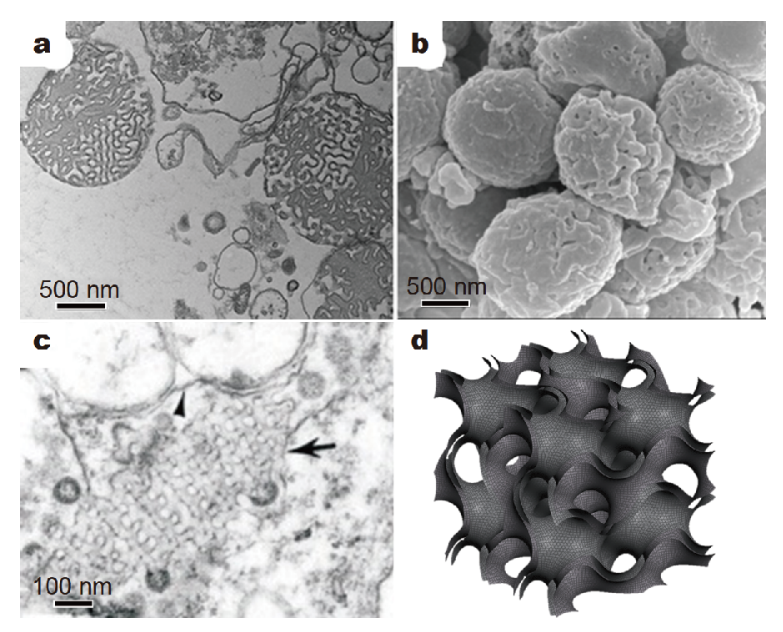

Figure 2 (a) Isolated mitochondria from 7-day starved amoeba Chaos carolinense revealed by TEM. (b) SEM micrograph showing the intact outer surfaces of the isolated mitochondria. Reproduced with permission from Ref. [71]. Copyright 2014, Springer-Verlag Wien. (c) SARS$\mathrm{CoV}$-induced cubic membranes in Vero cell. Reproduced under the terms of the CC BY 4.0 License [74]. Copyright 2004, The Authors. (d) 3D model of gyroid surfaces.

a unit cell parameter $\sim 50 \mathrm{~nm}$ in size, which was interpreted as intersection bilayer stacking. However, this structure was later revealed to be a tetragonal deformation of the $\mathrm{P}$ surface (tP) [70]. The bicontinuous $\mathrm{P}$ surface structure is believed to show some advantages in gas exchange due to its interconnected framework with an open channel system.

It is noteworthy that not only a large number of singlebilayer and double-bilayer cubic membrane structures have been found, but multilayer cubic membranes also exist. The inner segment of the retinal cone photoreceptors of the common tree shrew (Tupaia glis) and northern tree shrew (Tupaia belangeri) contain several layers of mega-sized mitochondria. The inner membrane of these mitochondria forms a gyroid cubic membrane structure with an 8 to 12 bilayer membrane. (Fig. $3 \mathrm{a}_{1-3}$ ) $[54,75]$ This is the most complex cubic membrane structure ever discovered. These multilayer gyroid cubic membrane structures are capable of forming multiple focal points and blocking ultraviolet (UV) light independent of angle [12].

Furthermore, unbalanced CMC surface/parallel surface structures also exist in biological systems, specifically the biophotonic crystals discovered in certain insects. Some butterflies (e.g., Callophrys rubi) generate a metallic green color in their wing scales by making a gyroid CMC scaffold (Fig. $3 b_{1-3}$ ) [7-9,36]. It has been discovered that the scaffold is formed by the deposition of chitin inside a

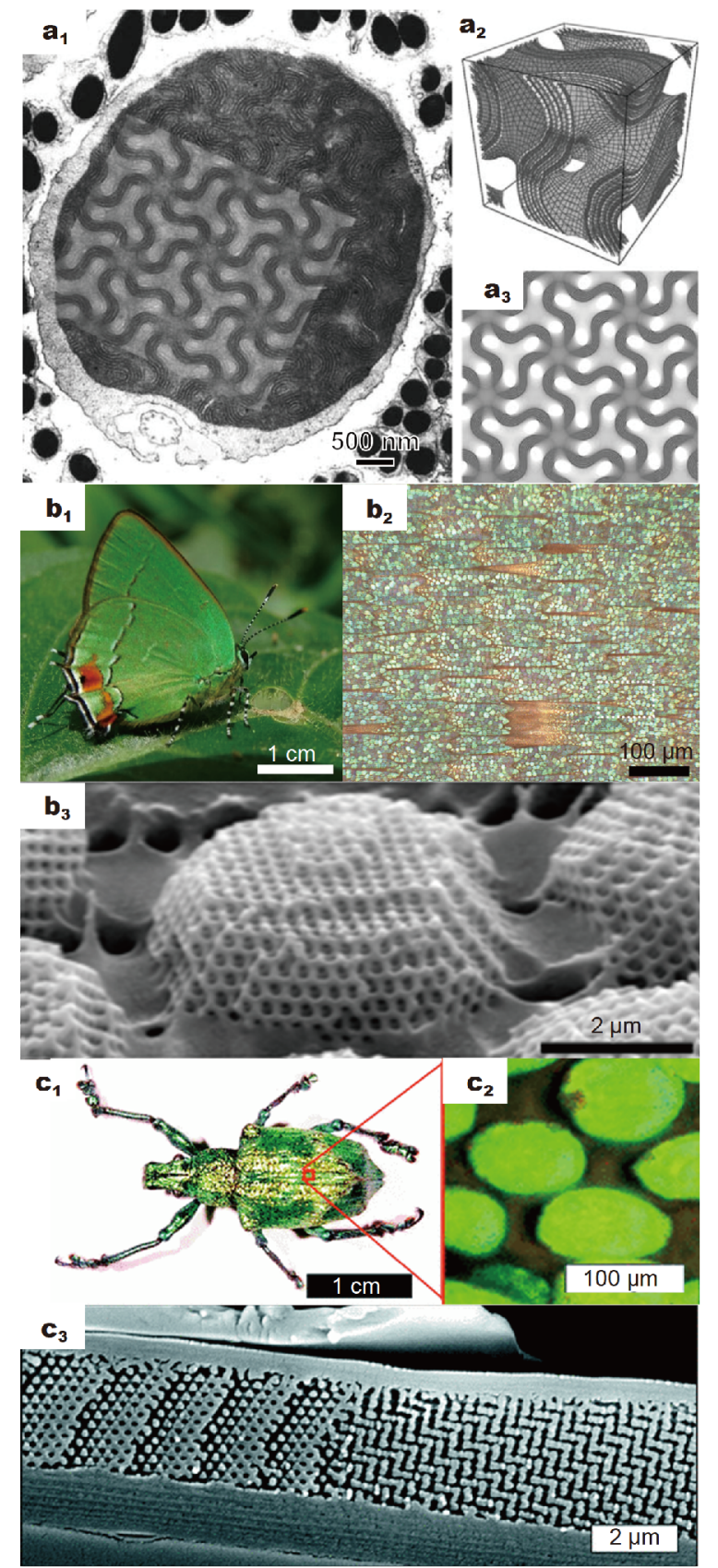

Figure $3\left(a_{1}\right)$ TEM images of mitochondria with multilayer cubic membranes in a tree shrew cone photoreceptor cell. $\left(\mathrm{a}_{2}\right) 3 \mathrm{D}$ model of 12layered $G$ surfaces and $\left(a_{3}\right)$ the corresponding $2 D$ projection map. The TEM image exactly matches the 2D simulated projection. Reproduced with permission from Ref. [75], Copyright 1987, Springer-Verlagl and Ref. [54], Copyright 2009, Elsevier. $\left(b_{1}\right)$ Photograph of T. opisena. $\left(b_{2}\right)$ Optical micrograph of the green ventral wing area. $\left(b_{3}\right)$ SEM image of SG crystallites. Reproduced under the terms of the CC BY 4.0 License [76]. Copyright 2017, The Authors. ( $\left.c_{1}\right)$ Photograph of the weevil $L$. augustus. $\left(\mathrm{c}_{2}\right)$ Optical micrograph of individual scales attached to the exoskeleton of L. augustus. $\left(c_{3}\right)$ Cross-sectioned SEM image of a region of a scale of L. augustus. Reproduced with permission from Ref. [11]. Copyright 2008, American Physical Society. 
convoluted membrane described by gyroid TPMS in the subcellular organelle of the endoplasmic reticulum (ER) [76]. As only one labyrinth of the gyroid TPMS was filled with chitin, the scaffold structure is single gyroid (SG). Similarly, a single diamond (SD) scaffold with a diamond CMC surface was identified in the exoskeletons of various beetles and weevils (Fig. $3 c_{1-3}$ ) $[10,11]$. Of note, both SG and SD structures are extraordinary 3D photonic crystal structures with complete bandgap properties. However, these photonic properties are absent in their corresponding double framework structures due to the increased symmetry [77].

\section{ARTIFICIAL SELF-ASSEMBLY OF AMPHIPHILIC MOLECULES}

On the other hand, TPMSs and their companions have been widely discovered in self-assembly systems containing amphiphilic molecules. For amphiphilic molecules with incompatible hydrophobic and hydrophilic components linked by covalent bonds, various types of LLC phases with periodic arrangements can be obtained depending on the geometric parameter $g=v / a_{0} l$ [78], where $v$ is the chain volume, $a_{0}$ is the effective hydrophobic/hydrophilic interfacial area, and $l$ is dynamical chain length. According to this scheme, spherical, cylindrical, TPMS, lamellae, and their corresponding inverse phases can be formed. Particularly, TPMSs can be formed when $g=2 / 3$ or $g>1$ for inverse phases. Work pioneered by Luzzati et al. [79] investigated bicontinuous LLC phases in the soap/water system, and the following studies dealing with lipidic LLC phases and other systems have extensively stimulated this research field $[1,80]$. Selfassembled structures can be regulated by changing the lipid type and adding different amphiphilic mixtures. Angelova et al. [81-86] studied the self-assembly of monoolein to form bicontinuous cubic phases in a mixture of different lipids or amphiphilic polymers. The effects of the additives on the packing of nonlamellar lipid monoolein have been recognized, and result in a change in its phase transition temperature. The change in the solubility of the additive with temperature has been shown to be due to the adjustable pore diameter via the expansion or contraction of water channels [86-88]. It has also been proposed that the formation of cubic nanostructures is related to the accumulation and fusion of curved bilayers into an ordered membrane structure $[89,90]$.

Compared to the LLC phases formed by lipid or surfactant molecules, the block copolymeric systems show great diversity due to the versatile design of blocks with various chemicals and physical properties. During the self-assembly of block copolymers, various mesostructures with complex morphologies can be formed by microphase separation $[19,20,25,91-96]$. This process is jointly driven by mixed enthalpy and mixed entropy $[19,97,98]$. The thermodynamic equilibrium of the linear $\mathrm{AB}$ diblock copolymer self-assembled morphology depends on the volume fraction of each block $\left(f_{\mathrm{A}}\right.$ or $f_{\mathrm{B}}, f_{\mathrm{A}}+$ $\left.f_{\mathrm{B}}=1\right)$, the Flory-Huggins parameter $(\chi)$, and the total degree of polymerization $(N)$ [19]. $\chi$ is usually a small positive value and varies inversely with temperature when there is no strong interaction (e.g., hydrogen bonding) [91]. The segregation product of the $\chi N$ value determines the degree of microphase separation of the two blocks at a given temperature. As the temperature increases, $\chi N$ decreases to the weak segregation limit $(\chi N<10)$ and the copolymer becomes disordered (i.e., homogeneous). When the $\chi N$ is large enough, the copolymer is in a strong segregation limit $(\chi N \gg 10)$ to enable microphase separation [97,99-101]. The morphological changes in copolymer self-assembly are a function of $\chi N$ and $f_{\mathrm{A}}$ and can be predicted using the self-consistent mean-field theory $[24,102,103]$. The double gyroid is found to be stable in a narrow window between the cylindrical hexagonally phase and the lamellar phase. The experimental phase diagram [104] of the polyisoprene- $b$-polystyrene (PI-PS) diblock copolymer bulk is similar to the theoretical calculation (Fig. 4) [24]. The experimental phase diagram is not completely symmetrical because the molecular shape of isoprene and styrene are different, and the interactions are also different.

The $\mathrm{ABC}$ triblock terpolymers extend the parameter space $\left(\chi_{\mathrm{AB}}, \chi_{\mathrm{AC}}, \chi_{\mathrm{BC}}, N, f_{\mathrm{A}}, f_{\mathrm{B}}, f_{\mathrm{C}}\right)$, resulting in the morphological complexity of self-assembly [19,105-109]. It is noteworthy that the bicontinuous cubic phases can be more precisely manipulated, and several unique phases have been obtained. Notably, a core-shell gyroid structure was discovered in the microphase separation of polyisoprene- $b$-polystyrene- $b$-polydimethylsiloxane (PI-PSPDMS) triblock terpolymer [110]. The two independent polydimethylsiloxane (PDMS) networks are encased in the polystyrene (PS) shell and separated by the continuous polyisoprene (PI) segment. These boundaries divided the space into five independent continuous domains, which is sometimes called "pentacontinuous" in polymer science. Considering the topology that only two identical gyroidal frameworks exist, it is reasonable to name this a "core-shell bicontinuous" structure. More interestingly, a new cubic phase has been discovered in the self-assembly of $\mathrm{ABC}$ triblock terpolymers such as 

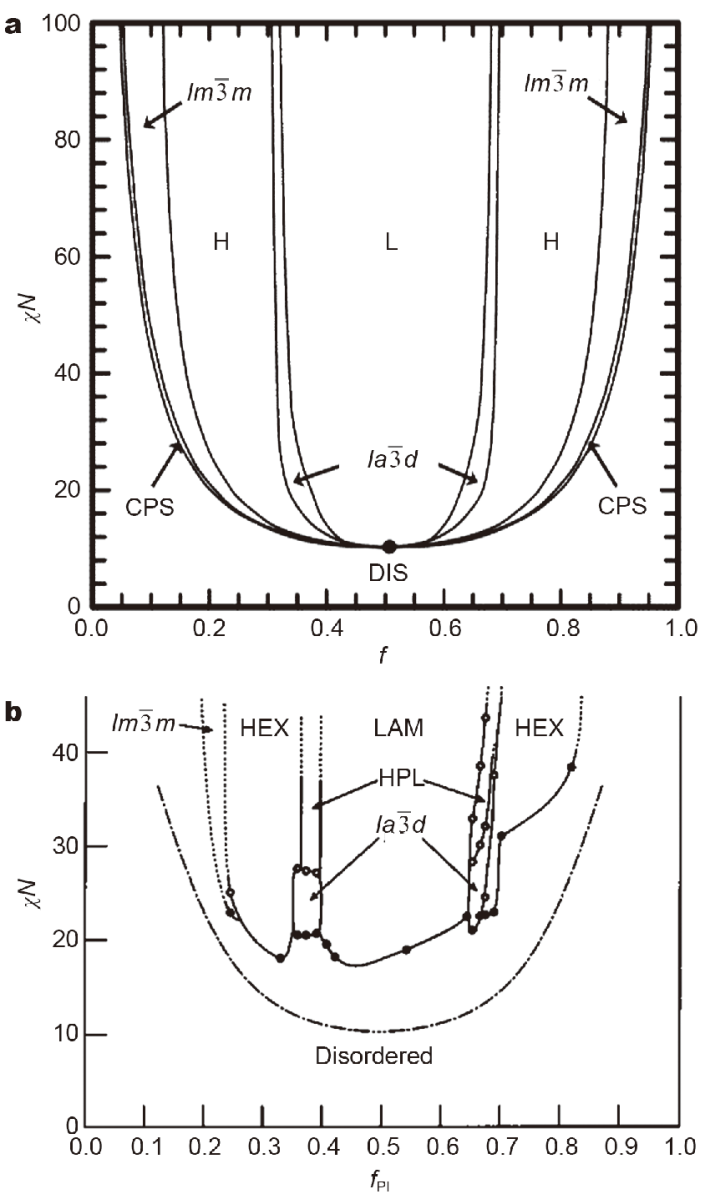

Figure 4 The phase diagram for linear $\mathrm{AB}$ diblock copolymers with theoretical calculation by (a) self-consistent mean-field theory and (b) experimental results for PI-PS. Phases are labeled lamellar (L, LAM), hexagonal cylinders (H, HEX), bicontinuous $I a-3 d$ cubic $(I a-3 d)$, bodycentered cubic (bcc) spheres (Im-3m), close-packed spheres (CPS), hexagonally perforated layers (HPL), and disordered (DIS). Reproduced with permission from Ref. [24], Copyright 2006, American Chemical Society and Ref. [104], Copyright 1995, American Chemical Society.

polyisoprene- $b$-polystyrene- $b$-poly(2-vinylpyridine) (PIPS-P2VP) and polyisoprene- $b$-polystyrene- $b$-polyethylene oxide (PI-PS-PEO) [37,111-114], which is recognized as an alternating gyroid $\left(\mathrm{Q}^{214}\right)$ [115-117]. In this phase, the B block forms the TPMS interface (matrix), while the $\mathrm{A}$ and $\mathrm{C}$ blocks separately form the two labyrinths with opposite handedness. As the $\mathrm{A}$ and $\mathrm{C}$ blocks are chemically distinguishable, the alternating gyroid is a useful technique for creating the thermodynamically unstable SG structure. This process can be performed either by infiltrating inorganic precursors into the polymer template, or by directly introducing the inorganic particles into the polymer self-assembly $[20,118,119]$.
In the above publications, the unit cell size of the polymer-based cubic phase structure is strongly dependent on the molecular weight of the polymer, and the typical unit cell parameter of the block polymer self-assembled is 5-100 $\mathrm{nm}$ [37]. To the best of our knowledge, the largest structure in the equilibrium microphase separation of block copolymer has a unit cell parameter of $258 \mathrm{~nm}$. The formation of such a large structure requires a two-week annealing treatment using a PS-PI block copolymer with a molecular weight of up to $750 \mathrm{~kg} \mathrm{~mol}^{-1}$ [120].

The microphase separation can be also tuned by adding solvent. A selective solvent (usually water) for one of the blocks of copolymer would drive copolymer aggregation into micelles via hydrophobic interactions. The morphology of the micelles is determined by the solvent selectivity $[121,122]$ and the composition of the copolymer $[123,124]$. As the volume fraction of the hydrophilic block decreases, spherical, cylindrical, and/or vesicular micelles are favored. A crew-cut micelle can be observed when the hydrophobic block has a very large volume fraction [123126], especially cubosomes with an internal bicontinuous cubic structure [123-131]. Lin et al. [128] employed a simple polystyrene- $b$-poly(ethylene oxide) (PS-PEO) diblock copolymer to obtain cubosomes with $\mathrm{P}$ and $\mathrm{D}$ surface mesophases, after which the selective solvent water was added to the dioxane/dimethylformamide solvent mixture to induce self-assembly. By using block copolymers comprised of different block types and structures, cubosomes with different structures and functions can be obtained [129-137].

Swelling the block copolymer using solvent is an effective way of increasing the unit cell size and can introduce a new parameter for the controlled organization of block copolymers. Han et al. [38,39,138] reported a delicate method for the fabrication of bicontinuous scaffolds using an amphiphilic $\mathrm{AB}$ or $\mathrm{ABC}$ block copolymer, e.g., poly(ethylene oxide)- $b$-polystyrene (PEO-PS) or poly(ethylene oxide)- $b$-polystyrene- $b$-poly(tert-butyl acrylate) (PEO-PS-PtBA), and an inorganic precursor in a tetrahydrofuran (THF)/water mixed solvent. The block copolymer used in this method (Fig. 5) is highly soluble in the common solvent THF; however, microphase separation can be induced by the addition of a small amount of the selective solvent water. The hydrophobic block (PS or PtBA-PS) is dissolved in the THF-rich phase, whereas water is mainly present in the hydrophilic region (PEO), forming two water-rich cores with interwoven frameworks. With a large THF/water ratio, a structure containing two equivalent labyrinths with multilayer 


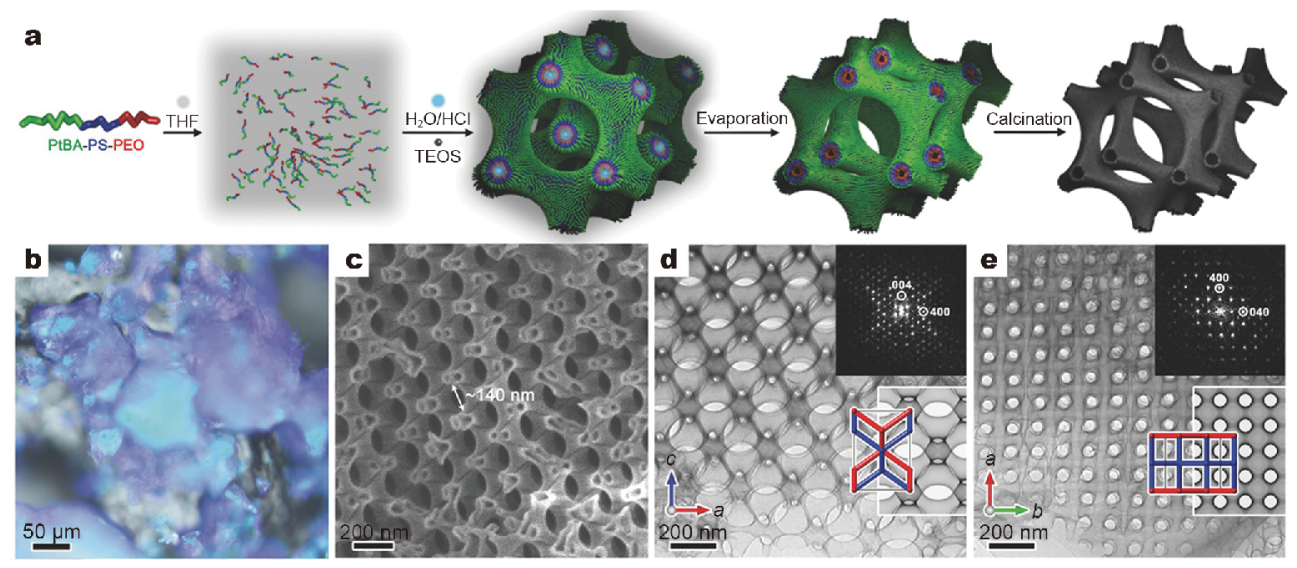

Figure 5 (a) Schematic diagram of the core-shell bicontinuous SDD structure formed by microphase separation with a mixture of solvents. TEOS: tetraethylorthosilicate. (b) Optical micrograph of the sample showing the structural color from purple to blue. (c) SEM and (d, e) TEM images with different orientations, showing two sets of hollow diamond frameworks adhering to each other. Reproduced with permission from Ref. [139], Copyright 2018, Wiley-VCH, and Ref. [38], Copyright 2014, The American Chemical Society.

core-shell bicontinuous double diamond (DD) framework is formed following the inverse phase (water-in-oil) configuration. Simultaneously, the silica precursor condenses in the hydrophilic regions to form the rigid silica scaffold. As the block copolymer exists only at the interface of THF-rich and water-rich phases, the entire structure can be swelled by solvents, and the size of the structure can thus be scaled up significantly. The unit cell parameter can be as large as $\sim 340 \mathrm{~nm}$ (original cubic unit cell) using a polymer with a relatively small molecular weight of $\sim 35.7 \mathrm{~kg} \mathrm{~mol}^{-1}$. Therefore, the unit cell parameters of the silica scaffold can go beyond the normal synthesis and are comparable with biological photonic structures [38]. Due to the large amount of THF that occupies space and separates the two interlocking double networks, the double networks shift and adhere each other to form a low-symmetry shifted DD (SDD) structure with the evaporation of the solvent, and this shifted structure introduces the photonic bandgap feature due to decreased crystal symmetry [38,138-140].

To understand the structural relationship and structural control in the self-assembly process, 108 triblock terpolymers PEO-PS-PtBA with different polymerization degrees have been synthesized to direct the formation of the inorganic scaffolds. Eight different structures have been obtained, including the normal-phase (oil-in-water) spherical type, normal-phase 2D hexagonal, lamellar, inverse-phase (water-in-oil) SDD, shifted double-primitive (SDP) and SG, and inverse-phase 2D hexagonal and inverse-phase spherical-type structures. The formation of these structures is dependent on the effect of the geometrical packing parameter determined by the volume fraction of the hydrophobic segment and the segregation product $(\chi N)$. Additionally, the different volume fraction ratios of the hydrophobic/hydrophilic block (PS/PEO, $\mathrm{PtBA} / \mathrm{PEO})$ and those of the hydrophobic/hydrophobic block (PS/PtBA) divide the resulting structures in several regions. It has been found that the formations of the bicontinuous structures are dependent on low volume fraction ratios of PS/PEO and PS/PtBA with similar packing parameters.

\section{SIMILARITIES AND DIFFERENCES BETWEEN BIOLOGICAL MEMBRANES AND ARTIFICIAL SYSTEMS}

\section{Structure and formation mechanism}

From a structural point of view, bicontinuous structures in biology and the artificial polymer system show high similarity. It is of great interest to determine whether this structural resemblance is due to a shared formation mechanism. LLC is only one of many important methods that nature employs to produce complex biological forms.

The formation of bicontinuous structures in LLC can be understood by the chain stretching and film bending associated with hydrophilic/hydrophobic interfaces, which lead to either a pair of CMC surface companions to the TPMS or a pair of parallel surfaces off the TPMS equidistant from the surface normal. The two surfaces correspond to the uniform curvature and the uniform chain length of the amphiphilic molecules [141-143]. However, the frustration present in most of the LLC phases is due to the nonuniformity of the curvature and chain length. Charvolin and Sadoc [144] discussed the 
curvature frustration in the formation of bicontinuous structures. Helfrich and Hyde $[145,146]$ proposed independently that the frustration in LLC corresponds to the fluctuations of the Gaussian curvature in TPMS. The stability of the bicontinuous structures has been further studied with regards to the balance between universal geometrical properties and the concentration constraints. After calculating the distribution of the Gaussian curvature, only G, D, and P surfaces were found to be stable phases among several TPMSs, and the G surface was the most stable [147,148]. Later, Schröder-Turk et al. [149] studied bicontinuous structures and molecular selfassembly by comparing the local curvature and the global packing homogeneity, which is defined as the standard deviation of the channel diameter of the labyrinths. The results confirmed that the $G$ surface is the most stable structure among the hyperbolic surface categories, followed by $\mathrm{D}$ and $\mathrm{P}$ surface structures. A recent publication indicated that bicontinuous phases are determined by the competition and compromise between the CMC and parallel surface models [150].

The formation of cubic membranes in nature is frequently associated with the overexpression of ER membrane-bound proteins. Lipids and proteins are not randomly distributed in biological membranes, and instead consist of a highly dynamic lipid raft and a stable submicron lipid domain [151-153]. These domains are composed of unique proteins and lipids [154-156]. Protein-protein and lipid-protein interactions, and/or lipid alterations, appear to play important roles in cubic membrane formation. In plants, it has been reported that the membrane domain of the enzyme 3-hydroxy-3-methylglutaryl coenzyme A (HMG-CoA) reductase is key for the proliferation of the ER and the formation of organized smooth endoplasmic reticulum (OSER) [157]. Additionally, it has been reported that the concentration of docosapentaenoic acid (DPA, C22:5n-6) in the cubic membrane of mitochondria in starved amoeba Chaos cells is about 1.6-fold higher than in fed cells. Under starvation conditions, the content of plasmalogen lipid is about 1.2 -fold that of fed cells $[158,159]$. The cubic membrane can be induced by over-expression of cytochrome b(5) tagged with a green fluorescent protein in COS-7 and CV-1 cells [160]. In this system, the formation of cubic membranes is considered to be the consequence of weak protein-protein interactions, since point mutations preventing these protein-protein interactions also prevent the formation of cubic membranes [160].

Because the biological membranes are a mixture of a large number of molecules in different forms, the $g$ value could not accurately predict their phase structures. In contrast, block polymers have the advantage of a clear molecular structure, and the molecule can be extensively designed. The phase morphology and geometric properties of block copolymers can be controlled by adjusting the block copolymer type, polymerization degree, additives, and environmental parameters to control the moduli and the spontaneous curvatures. As discussed above, the microphase separation is driven by incompatibilities between different blocks according to their chemical compositions. During the melting of block copolymers, microphase separation approaches thermodynamic balance after several rounds of heating and annealing, and the obtained structure represents the minimum thermodynamic free energy of the bulk system $[19,103,161]$. The self-assembly process needs to be completed over a long period of time, while relying on kinetic limitations can result in an unusual metastable state (e.g., the perforated lamellar phase) [162]. The introduction of a solvent can effectively increase the diffusion rate of the system. The self-assembly process of block copolymers in solution is more like the LLC system. Compared to the phases in bulk, the introduction of solvent increases the complexity of self-assembly (Fig. 6) $[40,163]$.

\section{Structural length scale and unit cell size}

Various structures with different length scales as well as different Gaussian curvatures can be generated from the same molecules in biological systems. The cubic membrane structure and unit cell parameters in different tissues can vary, from a $\mathrm{P}$ surface structure with unit cell parameter $a$ of $50 \mathrm{~nm}$ (tubular myelin of rabbit lung lavage) [70] to a multilayer $G$ surface structure with $a$ of $400 \mathrm{~nm}$ (mitochondria in retinal cones of tree shrew) [12], and even an SD structure with $a$ of $450 \mathrm{~nm}$ (exoskeleton of beetles) [11]. Nevertheless, this is very difficult to replicate in artificial fabrications, and the length scales of the cubic structures formed by the self-assembly of amphiphilic molecules are significantly smaller than those of cubic membranes in biology. The unit cell parameters in the polymer micelle system are difficult to adjust without changing the template molecules. In addition, the multilayer gyroid structure present in biological cubic membranes is also a huge challenge for polymer selfassembly systems to mimic.

Therefore, scaling artificial fabrication is a huge challenge encountered by scientists. It has been reported that inverse bicontinuous cubic phases can be formed when charged phospholipids are introduced, with the unit cell 


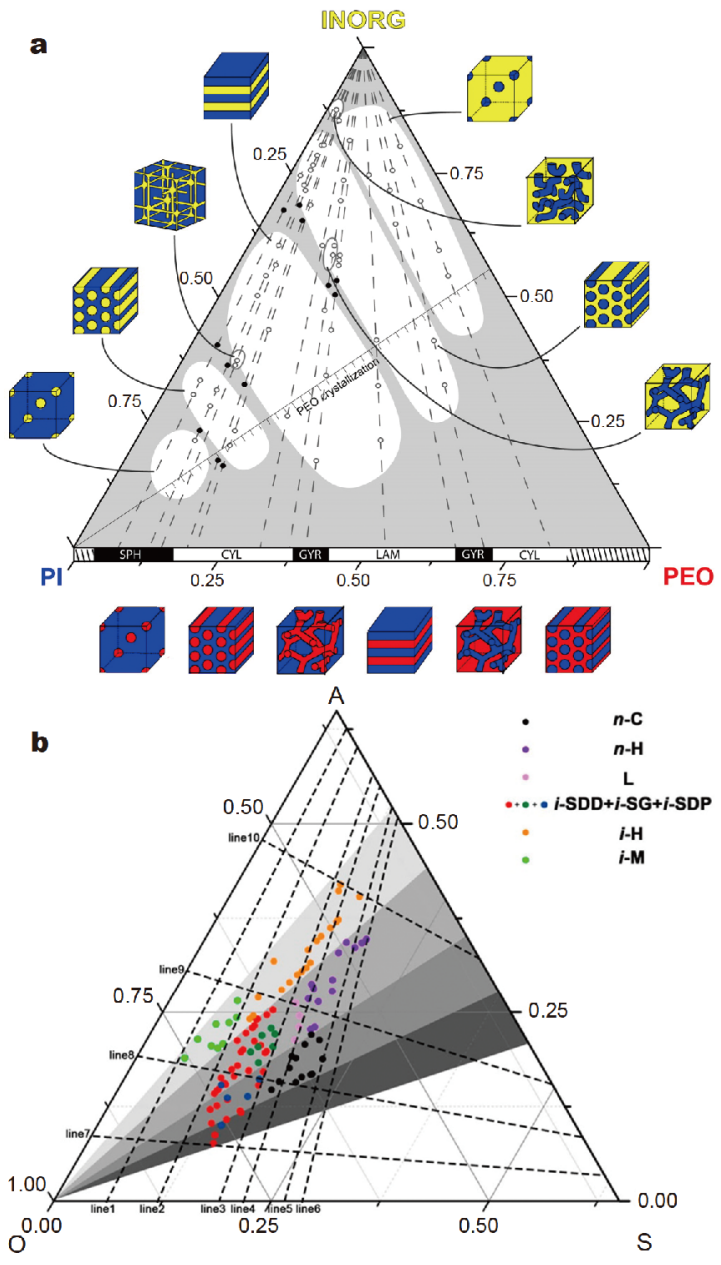

Figure 6 (a) Synthetic phase diagram of polyisoprene- $b$-poly(ethylene oxide) (PI-PEO) and inorganic components (in weight fractions). White areas are marked as different structures. A schematic of the phase of pure PI-PEO is shown at the bottom. Reproduced with permission from Ref. [163]. Copyright 2009, American Chemical Society. (b) Synthetic phase diagram of the structure of macroporous silica based on the PEOPS-PtBA polymer system (in volume fractions). Different points represent different structures: from normal-phase (oil in water) cage-type (n-C, black), normal-phase 2D hexagonal (n-H, purple), and lamellar ( $\mathrm{L}$, pink) to unique inverse-phase (water in oil) hyperbolic-surface (i-HS) structures, including the shifted double-diamond (i-SDD, red), singlegyroid (i-SG, dark green), and shifted double-primitive (i-SDP, blue), inverse-phase $2 \mathrm{D}$ hexagonal (i-H, orange) and inverse-phase micellar (iM, reseda). Reproduced with permission from Ref. [40]. Copyright 2018, American Chemical Society.

parameter increased from 10 to $50 \mathrm{~nm}$ [164-166]. Therefore, the formation of inverse micelles could be a promising method for creating materials containing a lattice size comparable to biological cubic membranes, especially those associated with structural colors.

The involvement of lipid and protein molecules with many different geometric parameters may be one of the reasons why biological cubic membranes have such large lattice and pore (channel) size parameters. As shown in the previous section, the typical unit cell size of the bicontinuous cubic phases is strongly dependent on the structural scale of the template molecules. For the LLCs formed by small molecules, the chain length plays a key role in channel size. For polymer-based cubic phases, the polymer chains are more extended in the structural size, the obtained structures are in the range of 5-100 nm, and block copolymers with extremely high molecular weight are required for a large unit cell parameter [37]. On the other hand, it has been recognized that the swelling of the cubic phase of lipid molecules can be achieved by the addition of different types of lipid molecules $[86,87]$. The effects of the polydispersity of block polymers on selfassembly behavior have also been recognized. Hustad et al. [167] obtained self-assembled mesophases with domain spacing over $100 \mathrm{~nm}$ using polydispersity olefin block copolymers with a total molecular weight below $100 \mathrm{~kg} \mathrm{~mol}^{-1}$. Nevertheless, the spacing of self-assembled block polymers with similar molecular weights is typically in the range of 30-50 nm. Kim et al. [168] reviewed the results of these experiments and calculations. The polydispersity of the block polymer can significantly increase the size of the domains during microphase separation. It can expand the accessible window of the gyroid cubic phase and the perforated lamellae.

Furthermore, the method of solvent swelling in the block copolymer region provides an effective means of adjusting the unit cell parameters of inverse bicontinuous cubic phases. Using this method, the block copolymer molecules stay only at the hydrophilic/hydrophobic interface and the space can be filled with a large amount of solvent. Typically, in the synthetic system of the reference [31], the unit cell parameter $a$ of the original cubic cell can be varied from 240 to $340 \mathrm{~nm}$ by adjusting the ratio of THF/water. As the unit cell parameters become larger, the SDD silica structure shows a partial band gap in the visible wavelength range, resulting in a structural color range ultraviolet to blue [38].

\section{Structural transition and structural determination}

In the study of biological bicontinuous phases, the defects and intergrowth of crystal structures are very important because they often provide information about the structural relationship in addition to the formation mechanism. The transformation of these structures has been extensively explored by several theoretical studies [149,169-172]. For membrane transformation, researchers generally believe that protein-driven changes in 
cell membrane curvature, the formation of stalks between the contacting monolayers, and vesicle fusion are the main reasons for biomembrane transformation [173176]. In this process, the stems are formed by transient contact between the membranes, which are then fused and expanded to form a tubular joint. These tubular connections constitute the basic connecting elements of bicontinuous cubic phases. Due to the sensitivity of biological membranes to external stimuli, these mechanisms cannot be easily observed or studied dynamically by experimentation. In addition, Norlén [177] proposed an intersection-free membrane-folding/unfolding model based on the formation of the mammalian skin barrier that merits attention. There is a continuous, dynamic film folding/unfolding process between cubic and lamellar structures in this model, and this process exists in many biological systems [178-180]. It does not involve budding or fusion or reducing energy consumption. At the same time, the continuity of the membrane can be maintained, which is essential for the barrier function of biological membranes. In addition, it is capable of forming parallel multilayer cubic membranes with large lattice parameters (50-500 nm).

These biological systems can be highly dynamic, coinciding with the nature of soft matters. It is extremely difficult to fully solve and characterize the structure by recognizing the $3 \mathrm{D}$ electrostatic potential map of the detailed structure in these biological systems. One of the key challenges in understanding their structure and structural transformation is experimental observation. Diffraction techniques such as X-ray diffraction (XRD) and small angle X-ray scattering (SAXS) have been widely used for the structural characterizations of LLC systems [181-184]. XRD and SAXS techniques provide overall information, but detailed morphological changes and intermediate structures cannot be easily revealed. TEM is a powerful technique that possesses certain advantages in its structural characterization abilities. TEM not only produces the electron diffraction patterns in reciprocal space, but also shows direct imaging in real space. Structural information can be obtained via 3D reconstruction of the target from a series of $2 \mathrm{D}$ projected images collected at incremental degrees of rotation around the specimen center. Furthermore, the structures can be identified based on pattern recognition through direct comparison with the experimental TEM images or the sliced samples with the $2 \mathrm{D}$ projection map generated by mathematical modeling with TPMS and related CMC/ parallel surfaces $[6,55,56]$.

The structural transformation of lipid and surfactant systems has been also extensively investigated by these technologies. Researchers have generated many phase diagrams for given lipids or mixture of lipids, describing the existence of various phase structures as a function of parameters such as composition, temperature, and pressure [183,185-187]. Meanwhile, LLC has a particular property that the phase structures can be fixed into inorganic frameworks. The mesostructures can be formed by cooperative self-assembly or true liquid crystalline template of the lipids or surfactants with the hydrolysis and condensation of inorganic precursors [188]. Various inorganic mesostructured materials can be produced according to the packing parameter $g$. The inorganic scaffolds can also be formed using amphiphilic block copolymers as templates [189-193]. Due to the stable inorganic framework, the electron crystallographic method can be applied to the structural investigation, and both the phases and amplitudes of crystal structure factors can be obtained from a Fourier transform of the high resolution TEM (HRTEM) images. Thus, a 3D electrostatic potential map can be directly reconstructed to elucidate characteristic structural features [194-196].

In the cooperative self-assembly of PEO-PS-PtBA and silica precursors, the epitaxial intergrowth of different hyperbolic surface structures has been discovered $[39,41]$. The "side-by-side" epitaxial relationship between SDD and SDP structures was identified. The $[001]_{\text {cub }}$ direction of SDP is parallel to the $[111]_{\text {cub }}$ direction of SDD (the subscript "cub" indicates the direction of the original cubic unit cell without shift), and the ratio of the unit cell parameters of double primitive (DP) to those of DD was 1.30 [39]. This is consistent with the theoretical value of 1.279 in Bonnet transformation, which keeps the Gaussian curvature unchanged (Fig. 7a, b). Furthermore, the intermediate interface was directly observed and found to be related to the rhombohedral deformation of $\mathrm{D}$ and $\mathrm{P}$ surfaces (rPD family) [149,171,172,183].

For the intergrowth of SDD and the thermodynamically unstable SG structure, a similar epitaxial relationship was confirmed, wherein the [001] of SG is parallel to the $[110]_{\text {cub }}$ of DD. Interestingly, the unit cell parameter of SG is only $\sqrt{2} / 2$ of the $\mathrm{DD}$, revealing the significance of the structural scale during the transition. Therefore, the nodes of both structures cannot be perfectly connected and the nodes in DD need to be branched to connect to that of the SG. Obviously, the structural transition is not a curvature-driven process, as there is a large lap in their Gaussian curvature. In order to explain the structural transformation, a modified alternating gyroid model was proposed, in which the TPMS is 


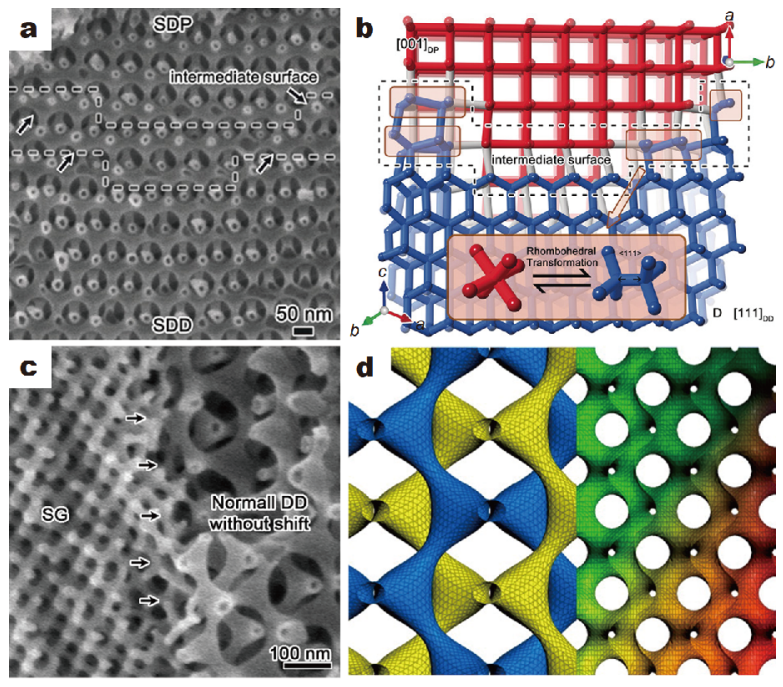

Figure 7 (a) SEM image of the intergrown structure of SDP and SDD. (b) Skeletal graph representing the centers of the channel for P to D networks according to SEM observations. Reproduced with permission from Ref. [39]. Copyright 2017, Wiley-VCH. (c) SEM images of the intergrown structure of DD and SG. (d) Schematic drawings of the structural relationships between the original DD and SG. Reproduced with permission from Ref. [41]. Copyright 2016, American Chemical Society.

formed by the hydrophobic part of the polymer while the two interwoven labyrinths are occupied by either the water-rich core or the THF-rich phase (Fig. 7c, d) [41].

It is noteworthy that the $2 \mathrm{D}$ projected TEM images of biological cubic membranes and macroporous silica formed by the block copolymer template show extremely high similarity, suggesting a 3D structural similarity. Interestingly, a similar structural transformation is also observed (Fig. 8). Biological membranes, as a complex, dynamic system, have a larger number of deformed phases, which are difficult to be accurately analyzed and characterized. Additionally, biological cubic membrane structures are often deformed and transformed from other structures, especially lamellar structures, so a large number of structural extension relationships exist $[197,198]$. After slicing the samples into ultra-thin sections, their structural features, including the structural transition and defects, can be seen using TEM. TEM is generally applied for cell ultrastructural studies and is also highly suitable for artificial materials synthesized with amphiphilic molecules. We managed to slice the intergrowth samples of DD and SG, which clearly show the structural transition of the epitaxial intergrowth of both structures via surface deformation and the connection of nodes with different Gaussian curvatures. These results demonstrate the possibility of exploring the structural
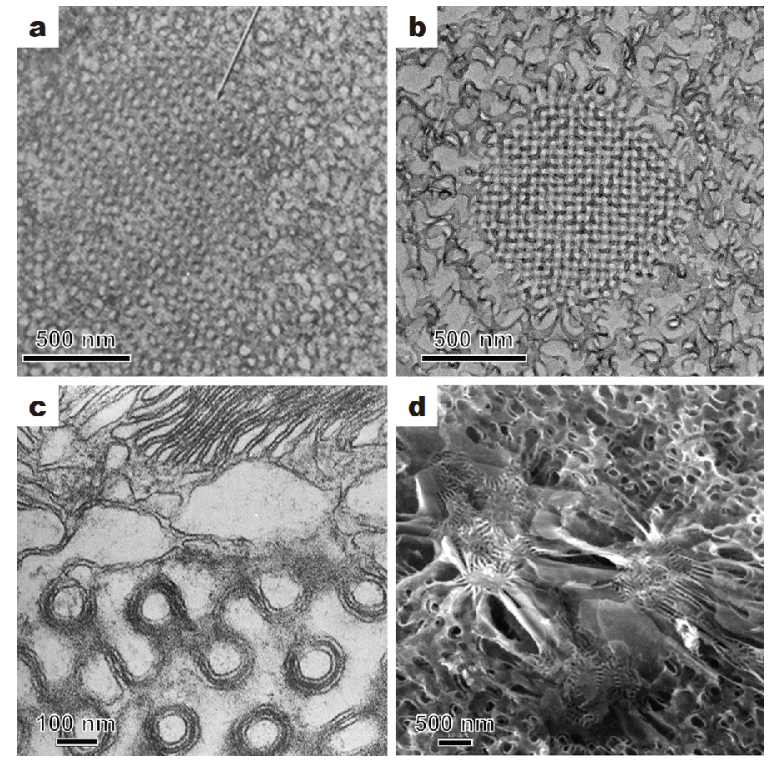

Figure 8 Structural transformations in biological membranes and artificial self-assembled systems. (a) ER in sebocytes of primates. Reproduced with permission from Ref. [197]. Copyright 1974, The Williams \& Wilkin Co. (b) SG structure in macroporous silica scaffolds. Intergrown/transformation between tubular and cubic structures in biological membranes (c) [198] and macroporous silica (d), respectively. Reproduced with permission from Ref. [198]. Copyright 1974, WileyVCH. The tubular and cubic membranes are connected by a sparse lamellar structure. They have almost the same transition patterns and behaviors in both systems. The SEM image in (d) has a stronger 3D effect and can show more details. (Images of $\mathrm{b}$ and $\mathrm{d}$ are provided by $\mathrm{Lu}$ Han.)

information and formation mechanism of cell membrane systems via TEM of the corresponding synthetic structures of the block copolymer system.

Importantly, kinetic control of the evaporation process yields some important and thermodynamically unstable intermediate phase structures. It may also provide a usable model and a powerful tool for investigating the complex structural transformation process of intracellular membrane systems. Because it directly reveals real space information, the analysis of electron crystallography possesses an advantage in studies of the defects and intergrowth of the mesophase structures.

\section{SUMMARY AND OUTLOOK}

Despite great achievements in this field in the past few decades, several challenges remain. As we have shown in this review, nature has many intelligent ways of producing complex biological bicontinuous structures. Some cubic membranes are spontaneously formed in cells, while others appear in response to external or internal stimuli. Researchers have found that there are many 
important physical and chemical properties of cubic cell membranes, including their interactions with biological macromolecules as well as optical and antioxidant properties, etc. However, their formation mechanisms and potential bionic applications are still poorly understood. Although the formation mechanism of cubic membranes in living organisms remains unclear, there must be a specific purpose for their formation aside from the simple geometric relationship of molecular packing. To address this question, experimental investigations conducted by mimicking the external and internal environments of the cells seem to be a good approximation. The difficulty lies in the complexity of the system, which is affected by numerous factors. Additionally, to understand their structure, an accurate description of their surface arrangement and the dynamic formation mechanism is essential. The advantages of contemporary cryo-TEM and environmental TEM may provide a deeper understanding of these cell membrane systems. Still, existing methods make it difficult to characterize and resolve fine details and dynamic structures in vivo.

In contrast, the formation of corresponding bicontinuous cubic phases in artificial polymer systems seems to be more straightforward. There are advantages to the well-defined, controllable, and designable molecular structure of artificial systems. The phase behavior of block copolymer systems with a stimulus responsive block can mimic biological membranes, and the formation of bicontinuous cubic phases may provide an ideal way to understand the much more complex cell membrane system. Specifically, electron microscopic techniques can be applied to the structural study of inorganic replicas of the LLC and copolymeric systems. Their stable inorganic framework makes a detailed structural study doable, particularly for the study of the structural transition, epitaxial growth, and crystal defects. Furthermore, the structure-determined functions in cell membrane systems can be deduced and studied using synthetic structures.

Learning from natural systems and bridging different research topics is key for the study of relevant structures. With this in mind, we attempted to compare the structural transformation and formation mechanisms of cell membrane systems and artificial systems by introducing the bicontinuous cubic phase in cell membranes and block copolymer systems. Therefore, the LLC and block polymer self-assembled system may serve as a suitable model system, wherein tunable synthesis and effective characterization methods can be fairly employed. Bridging the gap between the cell membrane system and the self-assembly process may provide new ideas for our understanding of the formation of cubic membranes, and this understanding may further guide approaches to synthesizing new functional materials. For instance, it remains a challenge to create a thermodynamically unbalanced single network CMC/parallel surface structure, which has been shown to possess excellent photonic properties. Additionally, in-depth study of the structure and properties of lipid bilayer membranes and soft materials is very valuable for the design and manufacture of biomimetic structural materials as stimuli responsive nanomaterials, drug delivery carriers, and hydrogels for regenerative medicine. By incorporating inorganic sources into the self-assembly system, novel functional materials may also be created with stable nano-network structures, enhanced functionalities, and expanded applications.

Received 19 November 2019; accepted 28 January 2020; published online 28 February 2020

1 Andersson S, Hyde ST, Larsson K, et al. Minimal surfaces and structures: From inorganic and metal crystals to cell membranes and biopolymers. Chem Rev, 1988, 88: 221-242

2 Hyde S, Blum Z, Landh T, et al. The Language of Shape: The Role of Curvature in Condensed Matter: Physics, Chemistry and Biology. Amsterdam: Elsevier, 1996

3 Donnay G, Pawson DL. X-ray diffraction studies of echinoderm plates. Science, 1969, 166: 1147-1150

4 Nissen HU. Crystal orientation and plate structure in echinoid skeletal units. Science, 1969, 166: 1150-1152

5 Larsson K, Fontell K, Krog N. Structural relationships between lamellar, cubic and hexagonal phases in monoglyceride-water systems. possibility of cubic structures in biological systems. Chem Phys Lipids, 1980, 27: 321-328

6 Landh T. From entangled membranes to eclectic morphologies: cubic membranes as subcellular space organizers. FEBS Lett, 1995, 369: 13-17

7 Michielsen K, Stavenga DG. Gyroid cuticular structures in butterfly wing scales: Biological photonic crystals. J R Soc Interface, 2008, 5: 85-94

8 Saranathan V, Osuji CO, Mochrie SGJ, et al. Structure, function, and self-assembly of single network gyroid (i4132) photonic crystals in butterfly wing scales. Proc Natl Acad Sci USA, 2010, 107: 11676-11681

9 Schröder-Turk GE, Wickham S, Averdunk H, et al. The chiral structure of porous chitin within the wing-scales of Callophrys rubi. J Struct Biol, 2011, 174: 290-295

10 Seago AE, Brady P, Vigneron JP, et al. Gold bugs and beyond: A review of iridescence and structural colour mechanisms in beetles (Coleoptera). J R Soc Interface, 2009, 6: S165

11 Galusha JW, Richey LR, Gardner JS, et al. Discovery of a diamond-based photonic crystal structure in beetle scales. Phys Rev E, 2008, 77: 050904

12 Almsherqi Z, Margadant F, Deng Y. A look through 'lens' cubic mitochondria. Interface Focus, 2012, 2: 539-545 
13 Luzzati V, Tardieu A, Gulik-Krzywicki T. Polymorphism of lipids. Nature, 1968, 217: 1028-1030

14 Scriven LE. Equilibrium bicontinuous structure. Nature, 1976, 263: $123-125$

15 Longley W, McIntosh TJ. A bicontinuous tetrahedral structure in a liquid-crystalline lipid. Nature, 1983, 303: 612-614

16 Fontell K. Cubic phases in surfactant and surfactant-like lipid systems. Colloid Polym Sci, 1990, 268: 264-285

17 Seddon JM. An inverse face-centered cubic phase formed by diacylglycerol-phosphatidylcholine mixtures. Biochemistry, 1990, 29: 7997-8002

18 Mackay AL. Periodic minimal surfaces. Nature, 1985, 314: 604606

19 Bates FS, Fredrickson GH. Block copolymers-designer soft materials. Phys Today, 1999, 52: 32-38

20 Orilall MC, Wiesner U. Block copolymer based composition and morphology control in nanostructured hybrid materials for energy conversion and storage: Solar cells, batteries, and fuel cells. Chem Soc Rev, 2011, 40: 520-535

21 Thomas EL, Anderson DM, Henkee CS, et al. Periodic areaminimizing surfaces in block copolymers. Nature, 1988, 334: 598601

22 Mai Y, Eisenberg A. Self-assembly of block copolymers. Chem Soc Rev, 2012, 41: 5969-5985

23 Hajduk DA, Harper PE, Gruner SM, et al. The gyroid: A new equilibrium morphology in weakly segregated diblock copolymers. Macromolecules, 1994, 27: 4063-4075

24 Cochran EW, Garcia-Cervera CJ, Fredrickson GH. Stability of the gyroid phase in diblock copolymers at strong segregation. Macromolecules, 2006, 39: 2449-2451

25 Stefik M, Guldin S, Vignolini S, et al. Block copolymer self-assembly for nanophotonics. Chem Soc Rev, 2015, 44: 5076-5091

26 Blum Z, Hyde S. On the templating of curvature in zeolites. Chem Mater, 1990, 2: 312-314

27 Sun J, Bonneau C, Cantín A, et al. The ITQ-37 mesoporous chiral zeolite. Nature, 2009, 458: 1154-1157

28 Nesper R, von Schnering HG. Periodic potential surfaces in crystal structures. Angew Chem Int Ed Engl, 1986, 25: 110-112

29 Bruinsma R. Elasticity and excitations of minimal crystals. J Phys II France, 1992, 2: 425-451

30 Fujita N, Terasaki O. Band structure of the P, D, and G surfaces. Phys Rev B, 2005, 72: 085459

31 Drummond CJ, Fong C. Surfactant self-assembly objects as novel drug delivery vehicles. Curr Opin Colloid Interface Sci, 1999, 4: 449-456

32 Shah JC, Sadhale Y, Chilukuri DM. Cubic phase gels as drug delivery systems. Adv Drug Deliver Rev, 2001, 47: 229-250

33 Ellens H, Siegel DP, Alford D, et al. Membrane fusion and inverted phases. Biochemistry, 1989, 28: 3692-3703

34 Siegel DP, Banschbach JL. Lamellar/inverted cubic (L. alpha/QII) phase transition in $\mathrm{N}$-methylated-dioleoylphosphatidylethanolamine. Biochemistry, 1990, 29: 5975-5981

35 Shearman GC, Khoo BJ, Motherwell ML, et al. Calculations of and evidence for chain packing stress in inverse lyotropic bicontinuous cubic phases. Langmuir, 2007, 23: 7276-7285

36 Wilts BD, Clode PL, Patel NH, et al. Nature's functional nanomaterials: Growth or self-assembly? MRS Bull, 2019, 44: 106-112

37 Meuler AJ, Hillmyer MA, Bates FS. Ordered network mesostructures in block polymer materials. Macromolecules, 2009, 42: 7221-7250
38 Han L, Xu D, Liu Y, et al. Synthesis and characterization of macroporous photonic structure that consists of azimuthally shifted double-diamond silica frameworks. Chem Mater, 2014, 26: 7020-7028

39 Mao W, Cao X, Sheng Q, et al. Silica scaffold with shifted "Plumber's Nightmare" networks and their interconversion into diamond networks. Angew Chem Int Ed, 2017, 56: 10670-10675

40 Cao X, Mao W, Mai Y, et al. Formation of diverse ordered structures in $\mathrm{ABC}$ triblock terpolymer templated macroporous silicas. Macromolecules, 2018, 51: 4381-4396

41 Cao X, Xu D, Yao Y, et al. Interconversion of triply periodic constant mean curvature surface structures: From double diamond to single gyroid. Chem Mater, 2016, 28: 3691-3702

42 Wen PC, Mahinthichaichan P, Trebesch N, et al. Microscopic view of lipids and their diverse biological functions. Curr Opin Struct Biol, 2018, 51: 177-186

43 Goñi FM. The basic structure and dynamics of cell membranes: An update of the Singer-Nicolson model. Biochim Biophys Acta (BBA) - Biomembranes, 2014, 1838: 1467-1476

44 Nicolson GL. The Fluid-Mosaic model of membrane structure: Still relevant to understanding the structure, function and dynamics of biological membranes after more than 40 years. Biochim Biophys Acta (BBA) - Biomembranes, 2014, 1838: 14511466

45 Sych T, Mély Y, Römer W. Lipid self-assembly and lectin-induced reorganization of the plasma membrane. Phil Trans R Soc B, 2018, 373: 20170117

46 Vereb G, Szöllosi J, Matkó J, et al. Dynamic, yet structured: The cell membrane three decades after the Singer-Nicolson model. Proc Natl Acad Sci USA, 2003, 100: 8053-8058

47 Tieleman DP, Marrink SJ, Berendsen HJC. A computer perspective of membranes: Molecular dynamics studies of lipid bilayer systems. Biochim Biophys Acta (BBA) - Rev Biomembranes, 1997, 1331: 235-270

48 Singer SJ, Nicolson GL. The fluid mosaic model of the structure of cell membranes. Science, 1972, 175: 720-731

49 Pearson RH, Pascher I. The molecular structure of lecithin dihydrate. Nature, 1979, 281: 499-501

50 Deplazes E, Poger D, Cornell B, et al. The effect of $\mathrm{H}_{3} \mathrm{O}^{+}$on the membrane morphology and hydrogen bonding of a phospholipid bilayer. Biophys Rev, 2018, 10: 1371-1376

51 Cullis PR, De Kruijff B. Lipid polymorphism and the functional roles of lipids in biological membranes. Biochim Biophys Acta (BBA) - Rev Biomembranes, 1979, 559: 399-420

52 Bretscher MS. Asymmetrical lipid bilayer structure for biological membranes. Nat New Biol, 1972, 236: 11-12

53 Chong K, Deng Y. The three dimensionality of cell membranes: Lamellar to cubic membrane transition as investigated by electron microscopy. Methods Cell Biol, 2012, 108: 319-343

54 Almsherqi ZA, Landh T, Kohlwein SD, Deng Y. Cubic membranes: The missing dimension of cell membrane organization. Int Rev Cell Mol Biol, 2009, 274: 275-342

55 Deng Y, Mieczkowski M. Three-dimensional periodic cubic membrane structure in the mitochondria of amoebae Chaos carolinensis. Protoplasma, 1998, 203: 16-25

56 Almsherqi ZA, Kohlwein SD, Deng Y. Cubic membranes: a legend beyond the Flatland* of cell membrane organization. J Cell Biol, 2006, 173: 839-844

57 Lučić V, Förster F, Baumeister W. Structural studies by electron tomography: From cells to molecules. Annu Rev Biochem, 2005, 
74: 833-865

58 Deng Y, Marko M, Buttle KF, et al. Cubic membrane structure in amoeba (Chaos carolinensis) mitochondria determined by electron microscopic tomography. J Struct Biol, 1999, 127: 231-239

59 Landh T. Cubic Cell Membrane Architectures: Taking Another Look at Membrane Bound Cell Spaces. Lund: Lunds Universitet. 1996

60 Almsherqi ZA, McLachlan CS, Mossop P, et al. Direct template matching reveals a host subcellular membrane gyroid cubic structure that is associated with sars virus. Redox Report, 2005, 10: $167-171$

61 Pyke K. Plastid Biogenesis and Differentiation. Berlin: Springer, 2007. 1-28

62 Gunning BES. The greening process in plastids. Protoplasma, 1965, 60: 111-130

63 Solymosi K, Aronsson H. Etioplasts and Their Significance in Chloroplast Biogenesis. Secondary Etioplasts and Their Significance in Chloroplast Biogenesis. Dordrecht: Springer, 2013. 39-71

64 Rascio N, Orsenigo M, Arboit D. Prolamellar body transformation with increasing cell age in the maize leaf. Protoplasma, 1976, 90: 253-263

65 Rascio N, Colombo PM, Vecchia FD, et al. Intrathylakoidal crystal appearance during the vital cycle of spinach chloroplasts. Protoplasma, 1985, 126: 153-157

66 Bennett J, Schwender JR, Shaw EK, et al. Failure of corn leaves to acclimate to low irradiance. Role of protochlorophyllide reductase in regulating levels of five chlorophyll-binding proteins. Biochim Biophys Acta (BBA) - Bioenergetics, 1987, 892: 118-129

67 Adamson H, Packer N, Gregory J. Chloroplast development and the synthesis of chlorophyll and protochlorophyllide in Zostera transferred to darkness. Planta, 1985, 165: 469-476

68 Whatley JM. Chloroplast development in primary leaves of Phaseolus vulgaris. New Phytol, 1974, 73: 1097-1110

69 Mezzenga R, Seddon JM, Drummond CJ, et al. Nature-inspired design and application of lipidic lyotropic liquid crystals. Adv Mater, 2019, 31: 1900818

70 Larsson M, Larsson K. Periodic minimal surface organizations of the lipid bilayer at the lung surface and in cubic cytomembrane assemblies. Adv Colloid Interface Sci, 2014, 205: 68-73

71 Chong K, Tan OLL, Almsherqi ZA, et al. Isolation of mitochondria with cubic membrane morphology reveals specific ionic requirements for the preservation of membrane structure. Protoplasma, 2015, 252: 689-696

72 Almsherqi Z, Hyde S, Ramachandran M, et al. Cubic membranes: A structure-based design for DNA uptake. J R Soc Interface, 2008, 5: $1023-1029$

73 Chong K, Almsherqi ZA, Shen HM, et al. Cubic membrane formation supports cell survival of amoeba Chaos under starvationinduced stress. Protoplasma, 2018, 255: 517-525

74 Goldsmith CS, Tatti KM, Ksiazek TG, et al. Ultrastructural characterization of SARS coronavirus. Emerg Infect Dis, 2004, 10: 320-326

75 Foelix RF, Kretz R, Rager G. Structure and postnatal development of photoreceptors and their synapses in the retina of the tree shrew (Tupaia belangen). Cell Tissue Res, 1987, 247: 287-297

76 Wilts BD, Apeleo Zubiri B, Klatt MA, et al. Butterfly gyroid nanostructures as a time-frozen glimpse of intracellular membrane development. Sci Adv, 2017, 3: e1603119

77 Maldovan M, Urbas AM, Yufa N, et al. Photonic properties of bicontinuous cubic microphases. Phys Rev B, 2002, 65: 165123

78 Israelachvili JN, Mitchell DJ, Ninham BW. Theory of self-assembly of hydrocarbon amphiphiles into micelles and bilayers. J Chem Soc Faraday Trans 2, 1976, 72: 1525-1568

79 Luzzati V. Biological significance of lipid polymorphism: The cubic phases. Curr Opin Struct Biol, 1997, 7: 661-668

80 Lynch ML, Spicer PT. Bicontinuous Liquid Crystals. New Jersey: CRC Press. 2005

81 Angelov B, Angelova A, Vainio U, et al. Long-living intermediates during a lamellar to a diamond-cubic lipid phase transition: A small-angle X-ray scattering investigation. Langmuir, 2009, 25 : 3734-3742

82 Angelov B, Angelova A, Mutafchieva R, et al. SAXS investigation of a cubic to a sponge $\left(\mathrm{L}_{3}\right)$ phase transition in self-assembled lipid nanocarriers. Phys Chem Chem Phys, 2011, 13: 3073-3081

83 Angelov B, Angelova A, Filippov SK, et al. Multicompartment lipid cubic nanoparticles with high protein upload: Millisecond dynamics of formation. ACS Nano, 2014, 8: 5216-5226

84 Angelova A, Ollivon M, Campitelli A, et al. Lipid cubic phases as stable nanochannel network structures for protein biochip development: X-ray diffraction study. Langmuir, 2003, 19: 69286935

85 Angelova A, Angelov B, Papahadjopoulos-Sternberg B, et al. Structural organization of proteocubosome carriers involving medium- and large-size proteins. J Drug Deliver Sci Tech, 2005, 15: $108-112$

86 Angelov B, Angelova A, Garamus VM, et al. Small-angle neutron and X-ray scattering from amphiphilic stimuli-responsive diamond-type bicontinuous cubic phase. J Am Chem Soc, 2007, 129: 13474-13479

87 Angelov B, Angelova A, Ollivon M, et al. Diamond-type lipid cubic phase with large water channels. J Am Chem Soc, 2003, 125: 7188-7189

88 Angelova A, Angelov B, Lesieur S, et al. Dynamic control of nanofluidic channels in protein drug delivery vehicles. J Drug Deliver Sci Tech, 2008, 18: 41-45

89 Angelov B, Angelova A, Papahadjopoulos-Sternberg B, et al. Detailed structure of diamond-type lipid cubic nanoparticles. J Am Chem Soc, 2006, 128: 5813-5817

90 Angelov B, Angelova A, Garamus VM, et al. Earliest stage of the tetrahedral nanochannel formation in cubosome particles from unilamellar nanovesicles. Langmuir, 2012, 28: 16647-16655

91 Dolan JA, Wilts BD, Vignolini S, et al. Optical properties of gyroid structured materials: From photonic crystals to metamaterials. Adv Opt Mater, 2015, 3: 12-32

92 Matsen MW. Effect of architecture on the phase behavior of ABtype block copolymer melts. Macromolecules, 2012, 45: 21612165

93 Luo M, Luo Y, Li X. Non-spherical polymersomes driven by directional aromatic interactions. Sci China Mater, 2018, 61: 437438

94 Zhang K, Zhao Z, Huang J, et al. Self-recoverable semi-crystalline hydrogels with thermomechanics and shape memory performance. Sci China Mater, 2019, 62: 586-596

95 Zhou P, Liang F, Liu Y, et al. Janus colloidal copolymers. Sci China Mater, 2015, 58: 961-968

96 Liu Z, Guo K, Zhao N, et al. Polysaccharides-based nanohybrids: Promising candidates for biomedical materials. Sci China Mater, 2019, 62: 1831-1836

97 Kim JK, Yang SY, Lee Y, et al. Functional nanomaterials based on 
block copolymer self-assembly. Prog Polym Sci, 2010, 35: 13251349

98 Patterson D, Robard A. Thermodynamics of polymer compatibility. Macromolecules, 1978, 11: 690-695

99 Helfand E, Wasserman ZR. Block copolymer theory. 4. Narrow interphase approximation. Macromolecules, 1976, 9: 879-888

100 Helfand E, Wasserman ZR. Block copolymer theory. 5. Spherical domains. Macromolecules, 1978, 11: 960-966

101 Helfand E, Wasserman Z. Block copolymer theory. 6. Cylindrical domains. Macromolecules, 1980, 13: 994-998

102 Matsen MW, Bates FS. Unifying weak- and strong-segregation block copolymer theories. Macromolecules, 1996, 29: 1091-1098

103 Matsen MW, Schick M. Stable and unstable phases of a diblock copolymer melt. Phys Rev Lett, 1994, 72: 2660-2663

104 Khandpur AK, Foerster S, Bates FS, et al. Polyisoprene-polystyrene diblock copolymer phase diagram near the order-disorder transition. Macromolecules, 1995, 28: 8796-8806

105 Abetz V, Stadler R. ABC and BAC triblock copolymers-morphological engineering by variation of the block sequence. Macromol Symp, 1997, 113: 19-26

106 Matsushita Y, Choshi H, Fujimoto T, et al. Preparation and morphological properties of a triblock copolymer of the $\mathrm{ABC}$ type. Macromolecules, 1980, 13: 1053-1058

107 Hadjichristidis N, Iatrou H, Pitsikalis M, et al. Linear and nonlinear triblock terpolymers. Synthesis, self-assembly in selective solvents and in bulk. Prog Polym Sci, 2005, 30: 725-782

108 Zheng W, Wang ZG. Morphology of ABC triblock copolymers. Macromolecules, 1995, 28: 7215-7223

109 Gröschel AH, Schacher FH, Schmalz H, et al. Precise hierarchical self-assembly of multicompartment micelles. Nat Commun, 2012, 3: 710

110 Shefelbine TA, Vigild ME, Matsen MW, et al. Core-Shell gyroid morphology in a poly(isoprene-block-styrene-block-dimethylsiloxane) triblock copolymer. J Am Chem Soc, 1999, 121: 84578465

111 Mogi Y, Kotsuji H, Kaneko Y, et al. Preparation and morphology of triblock copolymers of the ABC type. Macromolecules, 1992, 25: 5408-5411

112 Mogi Y, Mori K, Matsushita Y, et al. Tricontinuous morphology of triblock copolymers of the ABC type. Macromolecules, 1992, 25: 5412-5415

113 Epps TH, Cochran EW, Hardy CM, et al. Network phases in ABC triblock copolymers. Macromolecules, 2004, 37: 7085-7088

114 Epps TH, Cochran EW, Bailey TS, et al. Ordered network phases in linear poly(isoprene- $b$-styrene- $b$-ethylene oxide) triblock copolymers. Macromolecules, 2004, 37: 8325-8341

115 Matsen MW. Gyroid versus double-diamond in ABC triblock copolymer melts. J Chem Phys, 1998, 108: 785-796

116 Dotera T, Hatano A. The diagonal bond method: A new lattice polymer model for simulation study of block copolymers. J Chem Phys, 1996, 105: 8413-8427

117 Dotera T. Tricontinuous cubic structures in ABC/A/C copolymer and homopolymer blends. Phys Rev Lett, 2002, 89: 205502

118 Vignolini S, Yufa NA, Cunha PS, et al. A 3D optical metamaterial made by self-assembly. Adv Mater, 2012, 24: OP23-OP27

119 Robbins SW, Beaucage PA, Sai H, et al. Block copolymer selfassembly-directed synthesis of mesoporous gyroidal superconductors. Sci Adv, 2016, 2: e1501119

120 Urbas AM, Maldovan M, DeRege P, et al. Bicontinuous cubic block copolymer photonic crystals. Adv Mater, 2002, 14: 1850-1853
121 Yu Y, Zhang L, Eisenberg A. Morphogenic effect of solvent on crew-cut aggregates of apmphiphilic diblock copolymers. Macromolecules, 1998, 31: 1144-1154

122 Bhargava P, Zheng JX, Li P, et al. Self-assembled polystyreneblock-poly(ethylene oxide) micelle morphologies in solution. Macromolecules, 2006, 39: 4880-4888

123 Zhang L, Eisenberg A. Multiple morphologies and characteristics of "crew-cut" micelle-like aggregates of polystyrene- $b$-poly(acrylic acid) diblock copolymers in aqueous solutions. J Am Chem Soc, 1996, 118: 3168-3181

124 Zhang L, Eisenberg A. Formation of crew-cut aggregates of various morphologies from amphiphilic block copolymers in solution. Polym Adv Technol, 1998, 9: 677-699

125 Zhang L, Yu K, Eisenberg A. Ion-induced morphological changes in "crew-cut" aggregates of amphiphilic block copolymers. Science, 1996, 272: 1777-1779

126 Zhang L, Eisenberg A. Multiple morphologies of "crew-cut" aggregates of polystyrene- $b$-poly(acrylic acid) block copolymers. Science, 1995, 268: 1728-1731

127 Gröschel AH, Walther A. Block copolymer micelles with inverted morphologies. Angew Chem Int Ed, 2017, 56: 10992-10994

128 Lin Z, Liu S, Mao W, et al. Tunable self-assembly of diblock copolymers into colloidal particles with triply periodic minimal surfaces. Angew Chem Int Ed, 2017, 56: 7135-7140

129 La Y, Park C, Shin TJ, et al. Colloidal inverse bicontinuous cubic membranes of block copolymers with tunable surface functional groups. Nat Chem, 2014, 6: 534-541

130 Park C, La Y, An TH, et al. Mesoporous monoliths of inverse bicontinuous cubic phases of block copolymer bilayers. Nat Commun, 2015, 6: 6392

131 La Y, Song J, Jeong MG, et al. Templated synthesis of cubic crystalline single networks having large open-space lattices by polymer cubosomes. Nat Commun, 2018, 9: 5327

132 Jeong MG, Kim KT. Covalent stabilization of inverse bicontinuous cubic structures of block copolymer bilayers by photodimerization of indene pendant groups of polystyrene hydrophobic blocks. Macromolecules, 2016, 50: 223-234

133 Lyu X, Xiao A, Zhang W, et al. Head-tail asymmetry as the determining factor in the formation of polymer cubosomes or hexasomes in a rod-coil amphiphilic block copolymer. Angew Chem Int Ed, 2018, 57: 10132-10136

134 Azmi IDM, Moghimi SM, Yaghmur A. Cubosomes and hexosomes as versatile platforms for drug delivery. Therapeutic Deliver, 2015, 6: 1347-1364

135 Karami Z, Hamidi M. Cubosomes: Remarkable drug delivery potential. Drug Discovery Today, 2016, 21: 789-801

136 Yabu H. Self-organized precipitation: An emerging method for preparation of unique polymer particles. Polym J, 2013, 45: 261268

137 Grundy LS, Lee VE, Li N, et al. Rapid production of internally structured colloids by flash nanoprecipitation of block copolymer blends. ACS Nano, 2018, 12: 4660-4668

138 Li H, Liu Y, Cao X, et al. A shifted double-diamond titania scaffold. Angew Chem Int Ed, 2017, 56: 806-811

139 Han L, Che S. An overview of materials with triply periodic minimal surfaces and related geometry: From biological structures to self-assembled systems. Adv Mater, 2018, 30: 1705708

140 Sheng Q, Mao W, Han L, et al. Fabrication of photonic bandgap materials by shifting double frameworks. Chem Eur J, 2018, 24: 17389-17396 
141 Helfrich W. Elastic properties of lipid bilayers: Theory and possible experiments. Z für Naturforschung C, 1973, 28: 693-703

142 Sadoc JF, Charvolin J. Frustration in bilayers and topologies of liquid crystals of amphiphilic molecules. J Phys France, 1986, 47: 683-691

143 Anderson DM, Gruner SM, Leibler S. Geometrical aspects of the frustration in the cubic phases of lyotropic liquid crystals.. Proc Natl Acad Sci USA, 1988, 85: 5364-5368

144 Charvolin J, Sadoc JF. Periodic systems of frustrated fluid films and «bicontinuous» cubic structures in liquid crystals. J Phys France, 1987, 48: 1559-1569

145 Helfrich W, Rennschuh H. Landau theory of the lamellar-to-cubic phase transition. J Phys Colloques, 1990, 51: C7-189-C7-195

146 Hyde ST. Curvature and the global structure of interfaces in surfactant-water systems. J Phys Colloques, 1990, 51: C7-209-C7-228

147 Schwarz US, Gompper G. Stability of bicontinuous cubic phases in ternary amphiphilic systems with spontaneous curvature. J Chem Phys, 2000, 112: 3792-3802

148 Schwarz US, Gompper G. Stability of inverse bicontinuous cubic phases in lipid-water mixtures. Phys Rev Lett, 2000, 85: 1472-1475

149 Schröder-Turk GE, Fogden A, Hyde ST. Bicontinuous geometries and molecular self-assembly: Comparison of local curvature and global packing variations in genus-three cubic, tetragonal and rhombohedral surfaces. Eur Phys J B, 2006, 54: 509-524

150 Chen $\mathrm{H}$, Jin C. Competition brings out the best: Modelling the frustration between curvature energy and chain stretching energy of lyotropic liquid crystals in bicontinuous cubic phases. Interface Focus, 2017, 7: 20160114

151 Gronnier J, Gerbeau-Pissot P, Germain V, et al. Divide and rule: Plant plasma membrane organization. Trends Plant Sci, 2018, 23: 899-917

152 Simon KS, Pollock NL, Lee SC. Membrane protein nanoparticles: The shape of things to come. Biochem Soc Trans, 2018, 46: 14951504

153 Engelman DM. Membranes are more mosaic than fluid. Nature, 2005, 438: 578-580

154 Carquin M, D'Auria L, Pollet H, et al. Recent progress on lipid lateral heterogeneity in plasma membranes: From rafts to submicrometric domains. Prog Lipid Res, 2016, 62: 1-24

155 Krause MR, Regen SL. The structural role of cholesterol in cell membranes: From condensed bilayers to lipid rafts. Acc Chem Res, 2014, 47: 3512-3521

156 Sonnino S, Prinetti A. Membrane domains and the "Lipid Raft" concept. Curr Med Chem, 2013, 20: 4-21

157 Ferrero S, Grados-Torrez RE, Leivar P, et al. Proliferation and morphogenesis of the endoplasmic reticulum driven by the membrane domain of 3-hydroxy-3-methylglutaryl coenzyme a reductase in plant cells. Plant Physiol, 2015, 168: 899-914

158 Deng Y, Almsherqi ZA, Shui G, et al. Docosapentaenoic acid (DPA) is a critical determinant of cubic membrane formation in amoeba Chaos mitochondria. FASEB J, 2009, 23: 2866-2871

159 Lv WH, Liu FF, Deng YR. Biological cubic membranes. Prog Biochem Biophys, 2018, 45: 5-15

160 Snapp EL, Hegde RS, Francolini M, et al. Formation of stacked ER cisternae by low affinity protein interactions. J Cell Biol, 2003, 163: 257-269

161 Matsen MW, Bates FS. Origins of complex self-assembly in block copolymers. Macromolecules, 1996, 29: 7641-7644

162 Zhang L, Eisenberg A. Thermodynamic vs kinetic aspects in the formation and morphological transitions of crew-cut aggregates produced by self-assembly of polystyrene- $b$-poly(acrylic acid) block copolymers in dilute solution. Macromolecules, 1999, 32: 2239-2249

163 Garcia BC, Kamperman M, Ulrich R, et al. Morphology diagram of a diblock copolymer-aluminosilicate nanoparticle system. Chem Mater, 2009, 21: 5397-5405

164 Barriga HMG, Tyler AII, McCarthy NLC, et al. Temperature and pressure tuneable swollen bicontinuous cubic phases approaching nature's length scales. Soft Matter, 2015, 11: 600-607

165 Tyler AII, Barriga HMG, Parsons ES, et al. Electrostatic swelling of bicontinuous cubic lipid phases. Soft Matter, 2015, 11: 32793286

166 Zabara A, Chong JTY, Martiel I, et al. Design of ultra-swollen lipidic mesophases for the crystallization of membrane proteins with large extracellular domains. Nat Commun, 2018, 9: 544

167 Hustad PD, Marchand GR, Garcia-Meitin EI, et al. Photonic polyethylene from self-assembled mesophases of polydisperse olefin block copolymers. Macromolecules, 2009, 42: 3788-3794

168 Kim I, Li S. Recent progress on polydispersity effects on block copolymer phase behavior. Polym Rev, 2019, 59: 561-587

169 Sadoc JF, Charvolin J. Infinite periodic minimal surfaces and their crystallography in the hyperbolic plane. Acta Crystlogr A Found Crystlogr, 1989, 45: 10-20

170 Benedicto AD, O'Brien DF. Bicontinuous cubic morphologies in block copolymers and amphiphile/water systems: mathematical description through the minimal surfaces. Macromolecules, 1997, 30: 3395-3402

171 Fogden A, Hyde ST. Continuous transformations of cubic minimal surfaces. Eur Phys J B, 1999, 7: 91-104

172 Schröder GE, Ramsden SJ, Fogden A, et al. A rhombohedral family of minimal surfaces as a pathway between the $\mathrm{p}$ and $\mathrm{d}$ cubic mesophases. Physica A-Statistical Mech its Appl, 2004, 339: 137-144

173 Kozlovsky Y, Efrat A, Siegel DA, et al. Stalk phase formation: Effects of dehydration and saddle splay modulus. Biophys J, 2004, 87: $2508-2521$

174 Siegel DP, Kozlov MM. The Gaussian curvature elastic modulus of $\mathrm{N}$-monomethylated dioleoylphosphatidylethanolamine: Relevance to membrane fusion and lipid phase behavior. Biophys J, 2004, 87: 366-374

175 Chernomordik LV, Kozlov MM. Protein-lipid interplay in fusion and fission of biological membranes. Annu Rev Biochem, 2003, 72: $175-207$

176 Chernomordik LV, Kozlov MM. Mechanics of membrane fusion. Nat Struct Mol Biol, 2008, 15: 675-683

177 Norlén L. Skin barrier formation: The membrane folding model. J Invest Dermatol, 2001, 117: 823-829

178 Pathak RK, Luskey KL, Anderson RG. Biogenesis of the crystalloid endoplasmic reticulum in UT-1 cells: Evidence that newly formed endoplasmic reticulum emerges from the nuclear envelope.. J Cell Biol, 1986, 102: 2158-2168

179 Oparka KJ, Johnson RPC. Endoplasmic reticulum and crystalline fibrils in the root protophloem of Nymphoides peltata. Planta, 1978, 143: 21-27

180 Deng YR, Landh T. The cubic gyroid-based membrane structure of the chloroplast in Zygnema (Chlorophyceae Zygnematles). Zool Stud, 1995, 34: 175-177

181 Oka T. Transformation between inverse bicontinuous cubic phases of a lipid from diamond to gyroid. Langmuir, 2015, 31: 11353-11359

182 Seddon JM, Templer RH, Warrender NA, et al. Phosphati- 
dylcholine-fatty acid membranes: Effects of headgroup hydration on the phase behaviour and structural parameters of the gel and inverse hexagonal (HII) phases. Biochim Biophys Acta (BBA) Biomembranes, 1997, 1327: 131-147

183 Squires AM, Templer RH, Seddon JM, et al. Kinetics and mechanism of the interconversion of inverse bicontinuous cubic mesophases. Phys Rev E, 2005, 72: 011502

184 Oka T. Small-angle x-ray crystallography on single-crystal regions of inverse bicontinuous cubic phases: Lipid bilayer structures and Gaussian curvature-dependent fluctuations. J Phys Chem B, 2017, 121: 11399-11409

185 Kulkarni CV, Tang TY, Seddon AM, et al. Engineering bicontinuous cubic structures at the nanoscale-the role of chain splay. Soft Matter, 2010, 6: 3191-3194

186 Conn CE, Ces O, Squires AM, et al. A pressure-jump time-resolved X-ray diffraction study of cubic-cubic transition kinetics in monoolein. Langmuir, 2008, 24: 2331-2340

187 Qiu H, Caffrey M. The phase diagram of the monoolein/water system: Metastability and equilibrium aspects. Biomaterials, 2000, 21: 223-234

188 Wan Y, Zhao Y. On the controllable soft-templating approach to mesoporous silicates. Chem Rev, 2007, 107: 2821-2860

189 Alberius PCA, Frindell KL, Hayward RC, et al. General predictive syntheses of cubic, hexagonal, and lamellar silica and titania mesostructured thin films. Chem Mater, 2002, 14: 3284-3294

190 Soler-Illia GJAA, Sanchez C, Lebeau B, et al. Chemical strategies to design textured materials: from microporous and mesoporous oxides to nanonetworks and hierarchical structures. Chem Rev, 2002, 102: 4093-4138

191 Yang P, Deng T, Zhao D, et al. Hierarchically ordered oxides. Science, 1998, 282: 2244-2246

192 Yang P, Zhao D, Margolese DI, et al. Generalized syntheses of large-pore mesoporous metal oxides with semicrystalline frameworks. Nature, 1998, 396: 152-155

193 Yang P, Zhao D, Margolese DI, et al. Block copolymer templating syntheses of mesoporous metal oxides with large ordering lengths and semicrystalline framework. Chem Mater, 1999, 11: 2813-2826

194 Sakamoto Y, Kaneda M, Terasaki O, et al. Direct imaging of the pores and cages of three-dimensional mesoporous materials. Nature, 2000, 408: 449-453

195 Ohsuna T, Sakamoto Y, Terasaki O, et al. TEM image simulation of mesoporous crystals for structure type identification. Solid State Sci, 2011, 13: 736-744

196 Willhammar T, Sun J, Wan W, et al. Structure and catalytic properties of the most complex intergrown zeolite ITQ-39 determined by electron crystallography. Nat Chem, 2012, 4: 188-194

197 Bell M. A comparative study of the ultrastructure of the sebaceous glands of man and other primates. J Invest Dermatol, 1974, 62: 132-143

198 Öhman P. Fine structure of the retinal pigment epithelium of the river lamprey (Lampetra fluviatilis, Cyclostomi). Acta Zoolog, 1974, 55: 245-253

Acknowledgements This work was supported by the National Natural Science Foundation of China (21922304, 21873072 and 31670841), Shanghai Rising-Star Program (17QA1401700) and Wenzhou Institute, University of Chinese Academy of Sciences (WIUCASQD2019005).

Author contributions Han L and Deng Y proposed the topic and outline of the manuscript. The manuscript was originally drafted by Cui
C, and further enriched by Han L and Deng Y. All the co-authors contributed to the discussion and refinement of the manuscript.

Conflict of interest The authors declare that they have no conflict of interest.

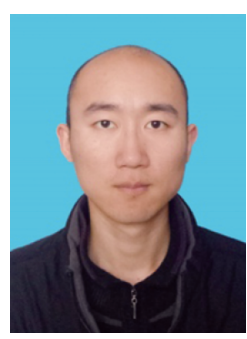

Congcong Cui received his Bachelor degree from Henan Normal University and Master degree from Yunnan Normal University. Currently, he is a research assistant in Tongji University. His research interest is focused on mesostructured materials and biomineralizations.

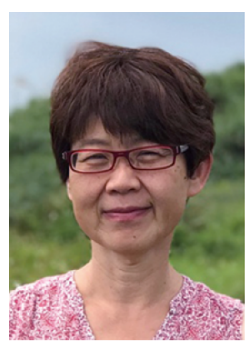

Yuru Deng received her undergraduate degree from Kaohsiung Medical University and $\mathrm{PhD}$ degree from State University of New York, USA. She did her postdoctoral training at Wadsworth Centre (NY), investigating Cubic Membrane (CM) nanostructures. She was an assistant professor at National University of Singapore (20022013) and is currently a Senior Research Associate at Wenzhou Institute, University of Chinese Academy of Sciences. She is internationally recognized as a pioneer in $\mathrm{CM}$ research, an emerging field in biomedicine and nano-technology.

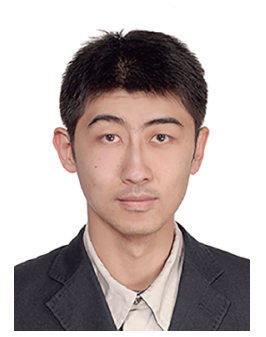

Lu Han received his undergraduate degree from Shanghai Jiao Tong University. He completed his $\mathrm{PhD}$ work in 2011 at Shanghai Jiao Tong University and at Stockholm University in 2010. He joined Shanghai Jiao Tong University as an assistant professor in 2011 and became an associate professor in 2013. He moved to Tongji University as a professor in 2017. His current research focuses on synthesis and characterization of mesostructured materials, biomineralizations, and structural analyses by transmission electron microscopy.

\section{生物和人工自组装体系中的双连续立方结构}

崔聪聪, 邓瑜如 ${ }^{2 *}$, 韩璐 ${ }^{{ }^{*}}$

摘要 在数十亿年的进化过程中, 大自然创造了多种复杂的多级结 构, 这些多级结构针对不同的生命活动具有组装体所不具有的优 异性能. 其中双连续立方结构通常能够用三周期极小曲面(TPMS) 和它们的常平均曲率曲面 (CMC)/平行曲面共同来描述. 它们具有 复杂的结构和独特的物理性质, 得到了各个研究领域的广泛关注, 同时也对它们的形成机理、晶体结构和性能表现的研究提出了新 的挑战. 本文对生物和人工自组装系统中相关曲面结构的形成机 理及相互关系进行了总结和讨论. 在生物体中具有几百纳米大尺 度的多种生物立方膜及相关结构, 而人工合成体系中往往只能形 成与分子大小及曲率相对应的结构. 值得注意的是, 嵌段共聚物体 系具有灵活的可设计性和丰富的相行为, 为研究生物体系提供了 一个理想的模型. 对这两个体系中发现的一些现象进行比较和讨 论, 可以为全面理解分子形状和由此产生的界面曲率之间的关系, 进而理解生物体的自组装过程提供新的思路. 